\title{
Correlação entre teores de nitrogênio e de clorofila na folha com o rendimento de grãos de aveia branca
}

\author{
Correlation among nitrogen and chlorophyll contents of leaves and grain yield of oat
}

\author{
William Messa Wolff ${ }^{\mathrm{I}}$ Elmar Luiz Floss $^{{ }^{*}}$
}

\section{RESUMO}

Um experimento foi conduzido na Universidade de Passo Fundo (UPF) com o objetivo de avaliar as correlações entre teores de $\mathrm{N}$ e de clorofila em folhas de aveia branca $e$ entre estes e o rendimento de grãos (RG), massa de mil grãos (MMG), período de enchimento (PEG) e taxa de enchimento de grãos (TEG). O ensaio foi conduzido no ano agrícola de 2003, utilizando-se 21 cultivares de aveia branca desenvolvidas pela FAMV/UPF, distribuídas em blocos ao acaso, em quatro repetições. Estimou-se o teor de clorofila por meio do índice SPAD (clorofilômetro) e determinou-se o seu teor pelo método químico e também os teores de $N$, nas folhas bandeia $e$ bandeira-1, no estádio de antese. Na maturação (colheita), determinou-se o RG, o MMG, o TEG e o PEG. Verificou-se que não houve correlações significativas entre teores de clorofila com os teores de N, com RG, MMG, TEG e PEG, porém, houve correlações significativas entre teores de $N$ com o RG, MMG e PEG.

Palavras-chave: clorofilômetro, massa de mil grãos, período de enchimento de grãos, taxa de enchimento de grãos.

\section{ABSTRACT}

An experiment was conducted at the Passo Fundo University, with the aim of evaluate the correlations among nitrogen contents, chlorophyll contents, grain yield (GY), thousand grain weight (TGW), grain filling period (GFP) and grain filling rate (GFR) in oat cultivars. The experiment was conducted during 2003 agriculture year, with oat cultivars developed by Passo Fundo University, with four replications, at random blocks distribution. The chlorophyll contents, estimated by chlorophyll meter (SPAD 502 model) and chemical method, and nitrogen contents were determined at anthesis stage. At maturation stage, the GY, TGW, GFP and GFR variables were determined. It was observed that there were no significant correlations among chlorophyll contents with nitrogen contents, with GY, TGW and GFP, but there were significant correlations among nitrogen contents and GY, TGW and GFP.

Key words: chlorophyll meter, thousand grain weight, grain filling period, grain filling rate.

\section{INTRODUÇÃO}

O nitrogênio é um dos elementos de maior importância na nutrição de plantas, pois é utilizado na síntese de compostos celulares, como a clorofila (LIMA et al., 2001). Nos cereais a adubação nitrogenada é baseada na recomendação da Comissão de Química e Fertilidade do Solo para o Rio Grande do Sul e Santa Catarina (COMISSÃO..., 2004). Entretanto, a adubação nitrogenada tem sido sub ou superestimada (ARGENTA et al., 2003), reduzindo a renda pela redução do rendimento de grãos ou pelo gasto desnecessário com fertilizante nitrogenado (ARGENTA et al., 2003). Há ainda os prejuízos ao ambiente, decorrentes da lixiviação de nitrato (WASKOM et al., 1996).

A agricultura de precisão possibilita a aplicação localizada de insumos agrícolas no local correto e nas quantidades requeridas (ARGENTA et al., 2001). É neste contexto que se enquadra o monitoramento do nível de $\mathrm{N}$, pela determinação do teor de clorofila na folha (ARGENTA et al., 2001).

A medição do teor de clorofila não é influenciada pelo consumo de luxo de $\mathrm{N}$, sob forma de nitrato (BLACKMER \& SCHEPERS, 1994), já que nesta

IFaculdade de Agronomia e Medicina Veterinária, Universidade de Passo Fundo (UPF), CP, 611, BR 285, Km 171, Bairro São José, 99001-970, Passo Fundo, RS, Brasil. E-mail: floss@upf.tche.br.*Autor para correspondência. 
forma o N não é assimilado na síntese da clorofila, não sendo quantificado na leitura do clorofilômetro (DWYER et al., 1995). Essa leitura (índice SPAD - Soil Plant Analisys Development) é considerada a melhor indicadora do estado nutricional de $\mathrm{N}$ na planta (BLACKMER \& SCHEPERS, 1994). O índice SPAD estima o teor relativo de clorofila, mediante valores calculados no aparelho a partir da quantidade de luz absorvida pela folha (WASKOM et al., 1996).

O objetivo do trabalho foi estimar os teores de clorofila (índice SPAD e método químico) e de N em folhas bandeia e bandeira-1 de aveia branca, bem como estabelecer correlações entre estes valores e entre os teores de clorofila e de $\mathrm{N}$ com o rendimento de grãos (RG), massa de mil grãos (MMG), período de enchimento de grãos (PEG) e taxa de enchimento de grãos (TEG).

\section{MATERIAL E MÉTODOS}

O ensaio foi conduzido em 2003, na Faculdade de Agronomia e Medicina Veterinária da Universidade de Passo Fundo (FAMV/UPF), em Latossolo Vermelho típico distrófico, unidade de mapeamento "Passo Fundo" (STRECK et al., 2002). O clima da região é classificado, segundo Köppen, como temperado-CFA(MORENO, 1961). A temperatura média anual é de $18,4^{\circ} \mathrm{C}$, a temperatura média das máximas é de $23,8^{\circ} \mathrm{C}$, e a temperatura média das mínimas é de $12,7^{\circ} \mathrm{C}$. A altitude é de $687 \mathrm{~m}$ acima do nível do mar (INSTITUTO..., 1989).

Foram avaliadas 21 cultivares de aveia branca desenvolvidas pela UPF, em quatro repetições, distribuídas em blocos ao acaso. Com base na análise de solo, foram aplicados $50 \mathrm{~kg} \mathrm{ha}^{-1}$ de $\mathrm{P}_{2} \mathrm{O}_{5}$ e $50 \mathrm{~kg} \mathrm{ha}^{-1}$ de $\mathrm{K}_{2} \mathrm{O}$, a lanço antes da semeadura, e $40 \mathrm{~kg} \mathrm{ha}^{-1}$ de $\mathrm{N}$ na forma amoniacal (uréia), no estádio de 3-4 folhas (COMISSÃO..., 2003).

As parcelas foram constituídas de sete linhas, com 5,0m de comprimento e $0,2 \mathrm{~m}$ de espaçamento entre linhas $\left(7,0 \mathrm{~m}^{2}\right)$. A semeadura direta foi realizada no dia 17 de junho de 2003, em sucessão à soja, com densidade de 300 sementes aptas por $\mathrm{m}^{2}$. Aos quinze dias após a emergência, realizou-se o desbaste, para a densidade uniforme de 250 plantas por $\mathrm{m}^{2}$.

O controle de plantas daninhas foi efetuado pela aplicação de bentazon $\left(1,5 \mathrm{~L} \mathrm{ha} \mathrm{h}^{-1}\right)$, seguido de capinas, quando necessário. $\mathrm{O}$ controle de moléstias foi efetuado mediante três aplicações de tebuconazole $\left(0,75 \mathrm{~L} \mathrm{ha}^{-1}\right)$, no aparecimento dos primeiros sintomas, e no seu ressurgimento. O controle de pragas foi efetuado pela aplicação de permetrina (200 $\left.\mathrm{mL} \mathrm{ha}^{-1}\right)$.
Os teores de clorofila foram estimados por dois métodos: índice SPAD e método químico, durante o estádio de antese. No campo, por meio do uso do medidor portátil de clorofila (clorofilômetro, modelo Minolta SPAD 502), determinou-se o índice SPAD nas folhas bandeira (CLFB) e folha bandeira-1 (CLFB-1). Após as leituras SPAD, as mesmas folhas foram coletadas para determinação do teor de clorofila pelo método químico (CQFB e CQFB-1), desenvolvido por KARAM (1998), com valores expressos em $\mu \mathrm{g}$ de clorofila por 0,1g de matéria fresca. O teor de N (NFB e NFB-1) foi determinado no Laboratório de Solos da FAMV/UPF, pelo método de Kjeldahl (MALAVOLTA et al., 1997).

A colheita dos grãos foi realizada por meio de colhedora de parcelas marca Wintersteiger. Após a colheita, os grãos foram despontados, limpos, pesados e foi determinado o teor de umidade. O rendimento de grãos (RG) foi estimado em $\mathrm{kg} \mathrm{ha}^{-1}$ a partir do peso de grãos de cada parcela $\left(1 \mathrm{~m}^{2}\right)$, corrigidos a $13 \%$ de umidade. O período de enchimento de grãos (PEG) corresponde ao número de dias da antese à maturação de colheita. A taxa de enchimento de grãos (TEG) foi calculada pela fórmula: TEG = RG/PEG. A massa de mil grãos (MMG) foi avaliada por meio da pesagem de 400 grãos escolhidos ao acaso, multiplicados por 2,5, expresso em g.

Efetuou-se a análise de variância pelo teste F e teste T de Student, pelo pacote SAS v. 6.12 (SAS, 1997). A comparação de médias pelo teste de Tukey e pelo teste $\mathrm{T}$ e as correlações entre as diferentes variáveis foram feitas em nível de 5\% de significância.

\section{RESULTADOS E DISCUSSÃO}

Na estimativa de teores de clorofila pelo clorofilômetro (CLFB e CLFB-1), determinação pelo método químico (CQFB e CQFB-1) e teores de $\mathrm{N}$ nas folhas bandeira e folha bandeira-1 (NFB, NFB-1), todas as variáveis apresentaram diferença significativa com exceção de CQFB (Tabela 1). Isso indica diferenças genotípicas quanto aos teores de $\mathrm{N}$ e de clorofila entre as cultivares, que confirmam os dados obtidos por McMULLAN et al. (1988), para a cultura da aveia.

Para as leituras SPAD, na folha bandeira, as cultivares com os maiores valores foram "UPF 19" $(52,8)$, “UPF 13” (52,5), “UPF 14” (51,6) e “UPF 11” (51,6), superando as cultivares "UPF 16" $(47,4)$, “UPF 18” (45,5), “UPF 7” (45,4) e “UPFA 22” - Temprana $(40,9)$, não diferindo das demais. Na folha bandeira-1, a cultivar “UPF 13” (51,5) foi superior às cultivares “UPF 2” $(46,1)$, “UPF 7” (43,4), “UPF 8” (46,0), “UPF 17” (43,5), “UPF 22” - Temprana (45,6) e “UPF 16” (42,6), não diferindo das demais. 
Tabela 1 - Clorofila determinada por leitura SPAD para folha bandeira (CLFB) e folha bandeira-1 (CLFB-1), clorofila determinada quimicamente para folha bandeira (CQFB) e folha bandeira-1 (CQFB-1), teor de N para folha bandeira (NFB) e folha bandeira-1 (NFB-1) no estádio de antese, para os cultivares de aveia-branca da UPF. FAMV/UPF, Passo Fundo/RS, 2003.

\begin{tabular}{|c|c|c|c|c|c|c|}
\hline Cultivar & CLFB (SPAD) & CLFB-1 (SPAD) & CQFB $(\mu \mathrm{g})$ & CQFB-1 $(\mu \mathrm{g})$ & NFB (\%) & NFB-1 (\% \\
\hline "UPF19" & A $52,8 a^{1}$ & B 47,2 abcd & NS 3,9 ns ${ }^{2}$ & $2,1 \quad$ cde & A 3,4 ab & B 2,7 ab \\
\hline “UPF13” & A 52,5 a & B 51,5 a & NS 3,0 & 3,4 abcde & A 3,3 abc & B $2,5 \mathrm{ab}$ \\
\hline “UPF14” & A 51,6 a & B 50,2 ab & NS 3,8 & 3,1 abcde & A 2,8 bcde & B $2,2 \mathrm{ab}$ \\
\hline “UPF11” & A 51,6 a & B 50,2 ab & NS 3,9 & 3,1 abcde & A 3,1 abcd & B $2,7 \mathrm{ab}$ \\
\hline “UPF6” & A $51,3 \mathrm{ab}$ & B 50,3 ab & NS 4,1 & $4,4 \mathrm{a}$ & A 3,0 abcde & B 2,6 ab \\
\hline “UPF15” & A $51,2 \mathrm{ab}$ & B 46,4 abcd & NS 3,9 & 3,8 abcd & A 3,3 abc & B 2,6 ab \\
\hline “UPF12” & A $51,0 \mathrm{ab}$ & B 48,4 abc & NS 3,6 & 3,6 abcde & A 3,2 abcd & B $2,5 \mathrm{ab}$ \\
\hline “UPF2” & A $51,0 \mathrm{ab}$ & B 46,1 bcd & NS 3,1 & 2,6 abcde & A 3,1 abcd & B 2,3 ab \\
\hline “UPF5” & A $50,5 \mathrm{ab}$ & B 48,3 abc & NS 4,0 & $4,3 \mathrm{ab}$ & A 3,2 abcd & B 2,6 ab \\
\hline “UPF10” & A 49,8 abc & B $48,4 \mathrm{abc}$ & NS 2,5 & 2,2 bcde & A 2,7 bcde & B 2,4 ab \\
\hline “UPF3” & B 49,8 abc & A $50,6 \mathrm{ab}$ & NS 3,7 & $3,8 \mathrm{abc}$ & A 3,1 abcd & B $2,7 \mathrm{ab}$ \\
\hline “UPF9” & A 49,7 abc & B 48,2 abc & NS 2,3 & 1,5 e & A 2,7 bcde & B $2,5 \mathrm{ab}$ \\
\hline “UPF1” & A $49,1 \mathrm{abc}$ & B 47,4 abcd & NS 3,7 & 2,2 bcde & A 3,1 abcd & B 2,6 ab \\
\hline "UPFA20” & B 49,0 abc & A $50,5 \mathrm{ab}$ & NS 3,6 & 3,0 abcde & A 3,6 a & B 2,8 a \\
\hline “UPF4” & A 48,5 abc & B $48,1 \mathrm{abc}$ & NS 3,0 & 2,6 abcde & A 3,2 abcd & B 2,4 ab \\
\hline “UPF8” & A 48,4 abc & B 46,0 bcd & NS 1,7 & 1,7 de & A 2,5 bcde & B $2,0 \quad b$ \\
\hline “UPF17” & A 48,3 abc & $\mathrm{B} 43,5 \mathrm{~cd}$ & NS 2,6 & 2,5 abcde & A 3,2 abcd & B 2,9 a \\
\hline “UPF16” & A 47,4 bc & $B \quad 42,6 \quad d$ & NS 3,2 & 1,6 e & A 3,3 abc & B 2,8 a \\
\hline “UPF18” & B 45,5 cd & A 46,5 abcd & NS 2,6 & 2,9 abcde & A 2,9 abcde & B 2,7ab \\
\hline “UPF7” & A 45,4 cd & B 43,4 cd & NS 3,2 & 1,8 cde & A $2,4 \quad$ e & B $2,0 \mathrm{~b}$ \\
\hline “UPFA22” & $\mathrm{B} 40,9 \quad \mathrm{~d}$ & A 45,6 bcd & NS 3,5 & 3,2 abcde & A 3,0 abcde & B 2,8 a \\
\hline Médias & 49,3 & 47,6 & 3,3 & 2,8 & 3,1 & 2,5 \\
\hline CV(\%) & 3,6 & 4,2 & 31,9 & 27,6 & 8,5 & 10,9 \\
\hline
\end{tabular}

1 - Médias seguidas de mesma letra minúscula na coluna e de mesma letra maiúscula na linha não diferem entre si pelo teste de Tukey e teste T de Student a 5\% de significância; 2 - ns = NS = não apresenta diferença significativa.

Em relação ao método químico, os teores de clorofila $(\mu \mathrm{g})$ na folha bandeira não apresentaram diferenças significativas entre cultivares. Na folha bandeira-1, a cultivar "UPF 6" $(4,4)$ foi superior às cultivares “UPF 1” (2,2), “UPF 7” (1,8), “UPF 8” $(1,7)$, “UPF 9” (1,5), “UPF 10” (2,2), “UPF 16” (1,6) e “UPF $19 ”(2,1)$, não diferindo das demais (Tabela 1$)$.

Ressalte-se que o coeficiente de variação obtido nas determinações de CQFB e CQFB-1 (31,9\% e $27,6 \%$, respectivamente) deve-se à complexidade da metodologia utilizada na determinação química (ARGENTA et al., 2001), aumentando a probabilidade de erros.

Para os teores de N (\%) da folha bandeira, a cultivar "UPFA 20" - Teixeirinha $(3,6)$ foi significativamente superior aos cultivares "UPF 7” $(2,4)$, “UPF 8” (2,5), “UPF 9” (2,7), “UPF 10” (2,7) e “UPF 14” $(2,8)$, não diferindo das demais. Quanto à folha bandeira1, as cultivares “UPF 16” (2,8), “UPF 17” (2,9), “UPFA 20” - Teixeirinha (2,8) e “UPFA22”-Temprana $(2,8)$ foram significativamente superiores às cultivares "UPF 7" $(2,0)$ e “UPF 8” (2,0), sem diferir das demais (Tabela 1).
Estes teores de $\mathrm{N}$ encontrados estão dentro do padrão encontrado por MENGEL \& KIRKBY (1982) e MARSCHNER (1995), para a cultura da aveia.

Comparando-se o teor de clorofila, não houve diferença significativa entre folha bandeira e bandeira-1, pelo método químico entre as cultivares, porém, pelo método do clorofilômetro, a folha bandeira apresentou valores SPAD significativamente superiores à folha bandeira-1, exceto para as cultivares “UPF 3”, “UPF 18”, “UPFA 20” -“Teixeirinha” e “UPFA 22” - Temprana, em que ocorreu o contrário. A semelhança estatística entre teores de clorofila nas folhas pelo método químico pode ser esplicado em virtude da dificuldade das determinações químicas (ARGENTA et al., 2001), mascarando os valores das variáveis. Já a superioridade estatística de teores de clorofila da FB sobre FB-1, pode ser atribuída ao fato de a leitura SPAD ser mais precisa que o método químico. Esta superioridade dos teores da FB em relação à FB-1 é confirmada por SMEAL \& ZHANG (1994), para o trigo, que observaram teores menores de clorofila em folhas mais velhas. Para os teores de $\mathrm{N}$, também 
houve diferença significativa, sendo que NFB foi superior à NFB-1 para todos as cultivares. Estes resultados são contrários aos de McMULLAN et al. (1988), que encontraram teores maiores de NFB-1 em relação a NFB para aveia .

Emrelação ao rendimento de grãos-RG $\left(\mathrm{kgha}^{-1}\right)$, a cultivar "UPFA 20" - "Teixeirinha” (4.236) foi superior às cultivares "UPF 7” (2.664), “UPF 13” (2.655), “UPF 10” (2.606), “UPF 8” (2.426) e “UPF 4” (2.033), sendo estatisticamente semelhante às demais. RIZZI (2004), também encontrou diferenças entre estes mesmas cultivares para o rendimento de grãos, nesta mesma magnitude (Tabela 2).

Para a massa de mil grãos-MMG (g), a cultivar “UPF 17” $(37,6)$, foi superior às demais, com exceção do cultivar “UPFA 20 - Teixeirinha” (36,4), correspondendo ao resultado encontrado por RIZZI (2004). A cultivar “UPFA 20 -Teixeirinha” não diferiu dos cultivares “UPF 15” (34,6), “UPFA 22" - Temprana $(33,8)$, “UPF 5” (34,3), e “UPF 17”.

Para o período de enchimento de grãos-PEG (dias), as cultivares recentemente desenvolvidas, como “UPF 16” (50,5), “UPF 17” e “UPFA 22” - Temprana (ambos com 50,3), apresentaram os maiores valores, diferindo significativamente das demais (mais antigos), com exceção das cultivares "UPFA 20 - Teixeirinha" $(47,5)$, “UPF 18” (46,8), ambos recentes, e “UPF 4” $(46,3)$. As cultivares que apresentaram os menores valores de PEG foram "UPF 7" $(32,3)$ e "UPF 8" $(30,8)$, ficando as demais cultivares, com exceção de "UPF 2" (35,8), “UPF 9” $(35,5)$ e “UPF 10” $(34,8)$ com valores intermediários. Estes resultados estão de acordo com BROUWER (1986), que destaca que cultivares mais recentes possuem maior PEG e com isso maior RG.

Em relação à taxa de enchimento de grãosTEG (kg ha-1 dia-1), a cultivar “UPF 19” $(96,0)$ foi superior à cultivar "UPF 4" $(43,4)$, não diferindo das demais cultivares. Entretanto, RIZZI (2004) não encontrou diferenças significativas para este caráter.

Quanto aos teores de N, ocorreram correlações positivas e significativas para NFB e NFB1 , com os valores de massa de mil grãos, período de enchimento de grãos e rendimento de grãos, mas não para taxa de enchimento de grãos (Tabela 3). Essa correlação entre teores de $\mathrm{N}$ e rendimento de grãos é destacada por PELTONEN-SAINIO (1994) e SLAFER (2004), que afirmam que a distribuição do N no dossel da cultura, para cereais, aumenta a eficiência na

Tabela 2 - Rendimento de grãos (RG), massa de mil grãos (MMG), período de enchimento de grãos (PEG) e taxa de enchimento de grãos (TEG) para as cultivares de aveia branca da UPF. FAMV/UPF, Passo Fundo/RS, 2003.

\begin{tabular}{|c|c|c|c|c|}
\hline Cultivar & $\mathrm{RG}\left(\mathrm{kg} \mathrm{ha}^{-1}\right)$ & MMG (g) & PEG (dias) & TEG $\left(\mathrm{kg} \mathrm{ha}^{-1} \mathrm{dia}^{-1}\right)$ \\
\hline "UPFA20" & $4.236 \mathrm{a}$ & $36,4 \mathrm{ab}$ & $47,5 \mathrm{ab}$ & 89,3 a \\
\hline “UPF18” & $4.054 \mathrm{ab}$ & 31,3 & 46,8 abc & 86,7 a \\
\hline “UPF16” & 3.965 abc & $32,2 \quad$ cdefg & 50,5 a & $77,4 \mathrm{ab}$ \\
\hline “UPF19” & 3.961 abc & 33,3 cdef & 41,3 cde & 96,0 a \\
\hline “UPF15” & 3.682 abc & 34,6 bc & 42,5 bcd & 86,8 a \\
\hline “UPFA22” & 3.630 abc & 33,8 bcde & 50,3 a & $72,2 \mathrm{ab}$ \\
\hline “UPF12” & 3.584 abcd & 31,5 & 43,5 bcd & $82,1 \mathrm{a}$ \\
\hline “UPF5” & 3.510 abcd & 34,3 bcd & 40,8 cdef & 86,2 a \\
\hline “UPF17” & $3.362 \mathrm{abcd}$ & 37,6 a & 50,3 a & $66,9 a b$ \\
\hline “UPF6” & $3.148 \mathrm{abcd}$ & 31,8 & 39,8 & 79,0 a \\
\hline “UPF2” & 3.008 abcd & 22,9 & 35,8 & $83,7 \mathrm{a}$ \\
\hline “UPF11” & 2.910 abcd & 30,7 & 39,8 def & $73,2 \mathrm{ab}$ \\
\hline “UPF9” & 2.818 abcd & 31,4 & 35,5 & 79,6 a \\
\hline “UPF1” & $2.807 \mathrm{abcd}$ & 27,1 & 35,5 & $79,0 \mathrm{a}$ \\
\hline “UPF14” & $2.767 \mathrm{abcd}$ & 30,3 & 39,8 & $69,6 \mathrm{ab}$ \\
\hline “UPF3” & $2.720 \mathrm{abcd}$ & 31,6 & 38,8 & $70,1 \mathrm{ab}$ \\
\hline “UPF7” & 2.664 bcd & 28,3 & 32,3 & 82,2 a \\
\hline “UPF13” & 2.655 bcd & 33,5 & 38,5 & $69,5 \mathrm{ab}$ \\
\hline “UPF10” & 2.606 bcd & 31,2 & 34,8 & $75,3 \mathrm{ab}$ \\
\hline “UPF8” & $2.426 \mathrm{~cd}$ & 27,6 & 30,8 & $79,1 \mathrm{a}$ \\
\hline “UPF4” & $2.033 \mathrm{~d}$ & 26,1 & 46,3 abc & $43,4 \quad b$ \\
\hline Médias & 3.169 & 31,3 & 41,0 & 77,5 \\
\hline $\mathrm{CV}(\%)$ & 18,7 & 3,3 & 5,7 & 16,7 \\
\hline
\end{tabular}

Médias seguidas de mesma letra na coluna não se diferenciam significativamente pelo teste de Tukey a 5\% de significância. 
Tabela 3 - Correlações entre rendimento de grãos (RG), massa de mil grãos (MMG), taxa de enchimento de grãos (TEG), período de enchimento de grãos (PEG) e teores de $\mathrm{N}$ na folha bandeira (NFB) e na folha abaixo da folha bandeira (NFB-1). FAMV/UPF, Passo Fundo/RS, 2003.

\begin{tabular}{lllll}
\hline Caracteres & MMG & PEG & RG & TEG \\
\hline NFB & $0,45^{*}$ & $0,63 * *$ & $0,53 *$ & $0,08 \mathrm{~ns}$ \\
NFB-1 & $0,66 * *$ & $0,75 * *$ & $0,64 *$ & $0,09 \mathrm{~ns}$ \\
PMG & 1 & $0,55 * *$ & $0,60 * *$ & $0,22 \mathrm{~ns}$ \\
TEG & $0,22 \mathrm{~ns}$ & $-0,17 \mathrm{~ns}$ & $0,66 * *$ & 1 \\
PEG & $0,55 * *$ & 1 & $0,63 * *$ & $-0,17 \mathrm{~ns}$ \\
\hline
\end{tabular}

ns - correlação não significativa a 5\% de significância pelo teste $\mathrm{T}$; * $\mathrm{P}<0.05$ pelo teste $\mathrm{T}$; $* *$ - $\mathrm{P}<0.01$ pelo teste $\mathrm{T}$.

interceptação de luz, aumentando o RG. WASKOM et al. (1996) e FLOSS et al. (1996) obtiveram correlações significativas entre teor de $\mathrm{N}$ e rendimento de grãos.

A massa de mil grãos-MMG correlacionase positivamente com teores de $\mathrm{N}$ na folha bandeira e bandeira-1, e, também com o período de enchimento de grãos e com o rendimento de grãos, ao contrário do que sugerem BROUWER (1986), SLAFER \& ANDRADE (1993) e SLAFER e MIRALLES (1993), que concluem que a tendência observada nas cultivares de maior RG é a estabilização ou a redução na MMG. Comparando-se os valores das correlações de NFB e NFB-1, observa-se que os valores de NFB-1 foram sempre superiores aos de NFB, o que pode significar que a FB-1 tem maior influência sobre estes caracteres avaliados, em relação à FB.

A duração do período de enchimento de grãos-PEG correlacionou-se positivamente com os teores de NFB $(0,63)$ e NFB-1 $(0,75)$, com a massa de mil grãos $(0,55)$ e com o rendimento de grãos $(0,63)$, confirmando a inter-relação entre estes caracteres. BROUWER (1986) e CALDERINI et al. (1995) destacam a contribuição do prolongamento do período de enchimento de grãos para o melhoramento de trigo no decorrer dos anos, que é associado com o maior rendimento e seus componentes.

A taxa de enchimento de grãos-TEG tem correlação significativa positiva com o rendimento $(0,66)$, mas não significativa com PEG $(-0,17)$, NFB $(0,08)$ e NFB-1 $(0,09)$, sugerindo que independe destes valores. A TEG influenciou significativamente o RG das cultivares avaliadas, cuja correlação positiva é confirmada por RIZZI (2004).

O rendimento de grãos-RG correlacionouse positivamente com NFB $(0,53)$, NFB-1 $(0,64)$, MMG $(0,60)$, PEG $(0,63)$ e TEG $(0,66)$ (Tabela 3). Estas correlações (MMG X RG e PEG X RG; NFB X RG e
NFB-1 X RG; TEG X RG) são confirmadas por BROUWER (1986), WASKOM et al. (1996) e RIZZI (2004), respectivamente.

\section{CONCLUSÕES}

As cultivares de aveia branca avaliadas não mostram correlações entre teores de clorofila (independente do método de determinação) e teores de $\mathrm{N}$, massa de mil grãos, período de enchimento de grãos, taxa de enchimento de grãos e rendimento de grãos. O teor de $\mathrm{N}$ na folha bandeira correlaciona-se positivamente com a massa de mil grãos, período e taxa de enchimento de grãos e rendimento de grãos. As cultivares mais recentemente desenvolvidas, de modo geral, apresentam maior eficiência do uso do N, quando comparadas às mais antigas.

\section{REFERÊNCIAS}

ARGENTA, G., et al. Clorofila na folha como indicador do nível de nitrogênio em cereais. Ciência Rural, v.31, p.715722, 2001.

ARGENTA, G. et al. Adubação nitrogenada em milho pelo monitoramento do nível de nitrogênio na planta por meio do clorofilômetro. Revista Brasileira de Ciência do Solo, v.27, p.109-119, 2003.

BLACKMER, T.M.; SCHEPERS, J.S. Techniques for monitoring crop nitrogen status in corn. Communications in Soil Science and Plant Analysis, v.25, p.1791-1800, 1994.

BROUWER, J.B. Crop physiological approaches to increased productivity in oats. In: INTERNATIONAL OAT CONFERENCE, 2, Dordrecht. Proceedings... Dordrecht: Martinus Niijhoff, 1986. p.149-159.

CALDERINI, D.F. et al. Genetic improvement in wheat yield and associated traits. A re-examination of previous results and the latest trends. Plant Breeding, v.114, p.108-112, 1995.

COMISSÃO BRASILEIRA DE PESQUISA DE AVEIA. Indicações técnicas para a cultura da aveia. Passo Fundo: Faculdade de Agronomia e Medicina Veterinária/UPF, 2003. 87p.

COMISSÃO DE QUÍMICA E FERTILIDADE DO SOLO - RS/ SC. Recomendações de adubação e calagem para os estados do Rio Grande do Sul e de Santa Catarina. Passo Fundo: SBCS - Núcleo Regional Sul, 2004.

DWYER, L.M. et al. Quantifying the nonlinearity in chlorophyll meter response to corn leaf nitrogen concentration. Canadian Journal of Plant Science, v.75, p.179-182, 1995

FLOSS, E.L. et al. Doses de nitrogênio em cobertura em aveia, sobre resteva de soja, 1995. In: REUNIÃO DA COMISSÃO SUL-BRASILEIRA DE PESQUISA DE AVEIA, 16., Florianópolis. Resultados experimentais. Florianópolis: UFSC, 1996 . p.302-305. 
INSTITUTO DE PESQUISA AGROPECUÁRIA. Seção de Ecologia Agrícola. Atlas Agroclimático do Estado do Rio Grande do Sul. Porto Alegre, 1989. 210p.

KARAM, D. Determinação de clorofila em tecido vegetal. Sete Lagoas: CNPMS - EMBRAPA, 1998. 5p.

LIMA, E. do V. et al. Adubação NK no desenvolvimento e na concentração de macro-nutrientes no florescimento do feijoeiro. Scientia Agrícola, v.58, p.125-129, 2001.

MALAVOLTA, E. et al. Avaliação do estado nutricional de plantas: princípios e aplicações. 2.ed. Piracicaba: POTAFOS, 1997. 319p.

MARSCHNER, H. Mineral nutrition of higher plants. 2.ed. London: Academic, 1995. 889p.

McMULLAN, P.M. et al. Dry matter and nitrogen accumulation and redistribution and their relationship to grain yield and grain protein in oats. Canadian Journal of Plant Science, p.983993, 1988.

MENGEL, K.; KIRKBY, E.A. Principles of plant nutrition. 3.ed. Switzerland: International Potash Institute, 1982. 655p.

MORENO, J.A. Clima do Rio Grande do Sul. Porto Alegre: Secretaria da Agricultura, 1961. 41p.

PELTONEN-SAINIO, P. Productivity of oats: genetic gains and associated physiological changes. In: SLAFER, G.A Genetic improvement of field crops. New York: Marcel Dekker, 1994. p.69-94.
RIZZI, S.P. Caracteres morfofisiológicos e produtividade de cultivares de aveia-branca. 2004. 87f. Dissertação (Mestrado em Agronomia/Produção Vegetal) - Curso de PósGraduação em Agronomia, Universidade de Passo Fundo.

SAS INSTITUTE. SAS/STAT software. Statistical Analysis System Institute. 1997.

SLAFER, G.A. Yield stability in cereals: past achievements and future progress. In: INTERNATIONAL OAT CONFERENCE, 7., 2004, Helsinki. Proceedings... Helsinki, 2004. p.57-63.

SLAFER, G.A.; ANDRADE, F.H. Physiological attributes related to the generation of grain yield in bread wheat cultivars released at different eras. Field Crops Research, v.31, p.351-367, 1993.

SLAFER, G.A., MIRALLES, D.J. Fruiting efficiency in three bread wheat (Triticum aestivum) cultivars released at different eras. Number of grains per spike and grain weight. Journal of Agronomy and Crop Science, v.170, p.251-260, 1993.

SMEAL, D.; ZHANG, H. Clhorophyill meter evaluation for nitrogen management in corn. Communications in Soil Science and Plant Analysis, v.25, n.9-10, p.1495-1503, 1994.

STRECK, E.V. et al. Solos do Rio Grande do Sul. Porto Alegre: EMATER/RS, UFRGS, 2002. 126p.

WASKOM, R.M. et al. Monitoring nitrogen status of corn with a portable chlorophyll meter. Communications in Soil Science and Plant Analysis, v.27, p.545-560, 1996. 